\title{
BIBLIOGRAPHY
}

1. J. Barta, Sulla risoluzione del problema di Dirichlet per il cerchio o per la sfera, Atti Acad. Naz. Lincei Mem. (6) 6 (1937), 783-793.

2. - Bornes pour la solution du problème de Dirichlet, Bull. Soc. Roy. Sci. Liège 31 (1962), 15-21.

3. - Sur une certaine formule qui exprime des bornes pour la solution du problème de Dirichlet, Bull. Soc. Roy. Sci. Liège 31 (1962), 760-766.

4. W. H. Malmheden, Eine neue Lösung des Dirichletschen Problems für sphärische Bereiche, Kung1. Fysiogr. Sällsk. i Lund Förh. 4 (1934), no. 17, 1-5. 111.

5. R. J. Duffin, A note on Poisson's integral, Quart. Appl. Math. 15 (1957), 109-

UNIVERSity OF MinNEsota

\section{A NOTE ON THE FUNDAMENTAL THEORY OF ORDINARY DIFFERENTIAL EQUATIONS}

BY GEORGE R. SELL ${ }^{1}$

Communicated by H. Antosiewicz, February 19, 1964

In this note we present some results on various problems connected with ordinary differential equations which do not necessarily satisfy a uniqueness condition. Using the concept of an integral funnel we are able to generalize the classical theorem on continuity with respect to initial conditions. This then leads to a reformulation of the problem of classifying the solutions of a given differential equation. That is, it is shown that every continuous vector field $f(x)$ on $W$ gives rise to a bicontinuous injection of $W$ into a space of functions $H$, and consequently the problem of classifying solutions is equivalent to the problem of characterizing this family of bicontinuous injections. A detailed discussion, with proofs, will appear later.

1. Introduction. Let us consider the differential equation

$$
x^{\prime}=f(x)
$$

where $f$ is defined and continuous on some open, connected set $W$ in $R^{n}$, real $n$-space. We shall let $W^{*}=W \cup\{\omega\}$ denote the one-point compactification of $W$. There is then at least one solution $\phi(p, t)$ of (1) through every point $p \in W$ with $\phi(p, 0)=p$. Moreover, every solution is defined on some maximal interval $J_{p}$ where either $J_{p}=R^{1}$ or $\phi(p, t) \rightarrow\{\omega\}$ as $t \rightarrow b d y J_{p}$. It should be noted that since the solutions of (1) may not be unique, the interval $J_{p}$ depends not only on $p$

\footnotetext{
1 The research on this paper was supported in part by a grant from the U. S. Army Research Office (Durham).
} 
but also on the particular solution $\phi(p, t)$.

Now define

$F(p, t)=F_{p}(t)=\{x \in W: x=\phi(p, t)$ for some solution of (1) through $p\}$.

If no solution of (1) can be continued up to time $t$, then $F(p, t)=\varnothing$, the empty set. If $A$ is any set in $W$, let

$$
F(A, t)=F_{A}(t)=\bigcup_{p \in A} F(p, t) .
$$

The set $F\left(A, R^{1}\right)=\mathrm{U}_{t \in R^{1}} F(A, t)$ is called the integral funnel through $A$, and each set $F(A, t)$ is said to be a section of the integral funnel.

The problem of characterizing the sets $F(p, t)$ and, more generally, $F(A, t)$ form the heart of the (local) fundamental theory of ordinary differential equations. There are, of course, two aspects to this problem. First, what topological properties do the sections $F(p, t)$ have and what topological properties of a given set $A$ in $W$ are inherited by the section $F(A, t)$. The second aspect is the converse of the above; that is, given a set $B$ in $W$, when is it the section of an integral funnel $F(p, t)$ ?

Under the assumption that $f(x)$ satisfies a local Lipschitz condition on $W$ or, more generally, that the solutions of (1) are unique, the first aspect of this problem has been solved. In this case, the mapping of $p \rightarrow F(p, t)$ is a homeomorphism of $A$ onto $F(A, t)$ provided $A$ is relatively compact in $W$ and $|t|$ is sufficiently small.

The converse question, even in the case where the solutions are unique, does not seem to be completely answered. We shall not consider this aspect of the problem here.

If the solutions of (1) are not unique, then the problem of characterizing the sets $F(p, t)$ and $F(A, t)$ naturally becomes more difficult. The first results on this problem are due to H. Kneser [7] who proved in 1923 that for $|t|$ sufficiently small, each section $F(p, t)$ is a continuum, that is, it is compact and connected. In the late 1920's, Fukuhara [2], [3] showed that if $q \in b d y F(p, \tau)$ for some $\tau$, then there is a solution $\phi(p, t)$ of $(1)$ which lies on $b d y F(p, t)$ for all $t$ between 0 and $\tau$ and $\phi(p, \tau)=q$. In 1932 Kamke [5] made a detailed investigation of the behavior of solutions of (1). He proved, among other things, that the sections $F(p, t)$ had a type of continuity in $p$. (See corollary to Theorem 4.) More recently various people have attempted an axiomatic approach to this problem. Of particular note is the work of Minkevič [8], [9], Barbašin [1] and Roxin [10].

Let $d(x, y)$ denote the Euclidean metric on $R^{n}$, then $|x|=\sqrt{ }(d(x, x))$ 
is the norm of $x$. If $A \subset R^{n}$ let $d(x, A)=\inf \{d(x, a): a \in A\}$. For every $\epsilon>0$ and every $A \subset R^{n}$, define $\mathfrak{B}(A, \epsilon)=\left\{x \in R^{n}: d(x, A)<\epsilon\right\}$ and $\mathfrak{D}(A, \epsilon)=\left\{x \in R^{n}: d(x, A) \leqq \epsilon\right\}$.

2. Basic properties of integral funnels. Let (1) be given on an open, connected set $W$ in $R^{n}$ and let $F(A, t)$ be the section of the integral funnel through $A$ where $A \subset W$. The following properties are easily established:

1. $F(A, 0)=A$.

2. If $t \tau \geqq 0$, then $F(A, t+\tau)=F(F(A, t), \tau)$.

3. If $t \tau<0$, then $F(A, t+\tau) \subset F(F(A, t), \tau)$.

Lemma 1. Let $A$ be a compact set in $W$ and let $\epsilon>0$ be given where $\mathfrak{D}(A, \boldsymbol{\epsilon}) \subset W$. Then there is an $\alpha>0$ such that $F(A, t) \subset \mathfrak{B}(A, \epsilon)$ for all $t \in(-\alpha, \alpha)$.

TheOREM 1. Let $A$ be a compact set in $W$. Then there is an $\alpha>0$ such that the section $F(A, t)$ is compact for every $t \in(-\alpha, \alpha)$.

If $A$ is a compact subset of $W$, then by Zorn's lemma there is an interval $I_{A}$ which is maximal with respect to the property that the section $F(A, t)$ is compact for every $t \in I_{A}$. (If $A=\{p\}$ we shall write $I_{A}$ as $I_{p}$.) It is clear that for each compact set $A$ in $W, 0$ is an interior point of $I_{A}$. Also, because of Theorem 1, $I_{A}$ is open.

3. General topological considerations. Since the sections $F_{p}(t)$ of the integral funnel are compact subsets of $W$, we can view $F_{p}$ as a function mapping $I_{p}$ into the compact subsets of $W$. Also, if $A$ is compact in $W$, then $F_{A}$ is a mapping of $I_{A}$ into the compact subsets of $W$.

Let $K=K(W)$ denote the collection of all nonempty compact subsets of $W$. We shall now define two topologies on $K$. The first, which we denote by $\mathcal{J}$, is defined in terms of a neighborhood basis for each point $A \in K$. Let $A \in K$ and let $\epsilon>0$ be given and define

$$
\mathfrak{N}(A, \epsilon)=\{B \in K: B \subset \mathfrak{B}(A, \epsilon)\} .
$$

Following Wilder's development [11], this neighborhood system generates a topology $J$ for $K$. It is easily shown that the neighborhoods themselves are open sets in this topology. Consequently, the family $\{\mathfrak{N}(A, \boldsymbol{\epsilon})\}$ of basic neighborhoods forms a basis for the topology.

The second topology on $K$, which we denote by $\mathfrak{H C}$, is the Hausdorff metric topology [4]. If $B$ and $A$ are in $K$, then we define

and

$$
\rho^{*}(A, B)=\inf \{\epsilon: B \in \mathfrak{N}(A, \epsilon)\}
$$




$$
\rho(A, B)=\max \left(\rho^{*}(A, B), \rho^{*}(B, A)\right) .
$$

$\rho$ is a metric on $K$ (the Hausdorff metric), and it generates the topology FC. Since $(K, \mathfrak{C})$ is a metric space, it is a normal Hausdorff space, or a $T_{4}$-space, in the terminology of Kelley [6]. However $(K, J)$ is a $T_{0}$ space, but it is not a $T_{1 \text {-space. }}$

Definition. Let $X$ be any topological space and let $g: X \rightarrow K$ be a function from $X$ into $K$. We shall say that $g$ is continuous if it is continuous in the J-topology on $K . g$ is said to be Hausdorff-continuous if it is continuous in the $\mathcal{H}$-topology on $K$. It is easy to check that Hausdorff-continuity implies continuity.

4. The function space $F$. Let $g$ be a continuous function from some interval $D_{g}$, which contains 0 , into $K$, and let $F$ denote the collection of all such continuous functions, where the domain $D_{g}$ depends on the function $g$. Before giving $F$ a topology we note the following facts.

Theorem 2. Let $A \in K$ and let $F_{A}$ and $I_{A}$ be given as above. Then $F_{A}(t)$ is Hausdorff-continuous on $I_{A}$, which implies that $F_{A} \in F$.

Theorem 3. Let $g \in F$ be defined on $D_{g}$ and let $J$ be a compact set in $D_{g}$. Then $g(J)=\bigcup_{t \in J} g(t)$ is a compact set in $W$.

We now construct a topology on $F$ by defining a neighborhood basis for each element of $F$. Let $g, J$ and $\epsilon$ be given where $g \in F, J$ is a compact set in $D_{g}$ and $\epsilon$ is a positive number. First define

$$
\mathfrak{R}(g, J, \epsilon)=\left\{h \in F: J \subset D_{h} \text { and } h(t) \in \mathfrak{N}(g(t), \epsilon) \text { for all } t \in J\right\} .
$$

Now define the neighborhood basis at $g$ by

$$
\mathfrak{M}(g, J, \epsilon)=\underset{0<\eta<\epsilon}{\bigcup} \mathfrak{R}(g, J, \eta) .
$$

This generates a topology on $F$, which we denote by $\mathfrak{T}$. Furthermore, the sets $\mathfrak{M}(g, J, \epsilon)$ are open in this topology, so they form a basis.

It is not hard to check that if $0<\eta<\epsilon$, then

$$
\mathfrak{R}(g, J, \eta) \subset \mathfrak{M}(g, J, \epsilon) \subset \mathfrak{R}(g, J, \epsilon)
$$

and that these inclusions are proper. Furthermore, one can show that, in general, the sets $\mathfrak{L}(g, J, \epsilon)$ are not open in the topology $\mathfrak{T}$. However, for Hausdorff-continuous functions we have:

Lemma 2. If $g \in F$ is Hausdorff-continuous, then $\mathbb{R}(g, J, \epsilon)$ is open and, in fact, $\mathfrak{M}(g, J, \epsilon)=\Re(g, J, \epsilon)$.

5. Continuity with respect to initial conditions. There are two subspaces of $F$ which are of particular importance for the study of the solutions of (1). These are 


$$
K=\left\{g \in F: g(t)=F_{A}(t) \text { on } I_{A} \text { for some } A \in K\right\}
$$

and

$$
W=\left\{g \in F: g(t)=F_{p}(t) \text { on } I_{p} \text { for some } p \in W\right\} .
$$

There is then a natural mapping from $K$ onto $K$, which we denote by

$$
G: A \rightarrow F_{A} \text {. }
$$

The restriction of $G$ to $W$ ( $W$ is viewed as a subspace of $K$ ) defines another mapping $H$ from $W$ onto $W$, where

$$
H: p \rightarrow F_{p}
$$

It is clear that the mappings $G$ and $H$ are one-to-one and onto. Consequently $G^{-1}$ and $H^{-1}$ exist.

The following theorem is the generalization of the classical continuity with respect to initial conditions.

Theorem 4. The mapping $G$ is a homeomorphism between $K$ and $K$, and $H$ is a homeomorphism between $W$ and $W$. Equivalently, $G$ and $H$ are bicontinuous injections of, respectively, $K$ and $W$ into $F$.

As a corollary we have the following result, which is a generalization of Kamke's theorem [5].

Corollary. Let $A \in K$ and let $J$ be a compact set in $I_{A}$. For every $\epsilon>0$ there is $a \delta>0$ such that if $q \in \mathfrak{B}(A, \delta)$, then $F(q, t) \subset \mathfrak{B}(F(A, t), \epsilon)$ $\subset \mathfrak{B}(F(A, J), \epsilon)$ for every $t \in J$.

6. Comments on the Hausdorff metric. Since the functions $F_{A}$ for $A \in K$ are Hausdorff-continuous, one could have restricted the discussion to the subspace $H$ of $F$ consisting of all Hausdorff-continuous functions. The mappings $G$ and $H$ are then bicontinuous injections of, respectively, $K$ and $W$ into $H$, where the topology on $H$ is generated by the neighborhood system $\{\mathfrak{M}(g, J, \epsilon) \cap H\}$.

It is natural to ask whether one could use the Hausdorff metric $\rho$ to define a different topology on $H$. For example, one could define a neighborhood basis for the function $g \in H$ as follows: Let $J$ be a compact set in $D_{0}$ and let $\epsilon>0$, then define

$$
\mathfrak{D}(g, J, \epsilon)=\left\{h \in H: \sup _{t \in J} \rho(h(t), g(t))<\epsilon\right\} .
$$

This generates a topology on $H$, which we now denote by $\mathcal{O}$. Furthermore the sets $\mathfrak{D}(g, J, \epsilon)$ are open and thus form a basis for the topol- 
ogy. If $g \in H$ it is easily checked that $\mathfrak{D}(g, J, \epsilon) \subset \mathfrak{M}(g, J, \epsilon) \cap H$ and that this inclusion is proper. Furthermore, the mappings $G^{-1}$ and $H^{-1}$ are continuous in the $\theta$-topology. However, the mappings $G$ and $H$ are generally not continuous in the $\theta$-topology, as is seen in the following example:

Let $W=R^{1}$ and let

$$
x^{\prime}=\left\{\begin{aligned}
\sqrt{ } x, & \text { if } x \geqq 0 \\
0, & \text { if } x<0 .
\end{aligned}\right.
$$

If $J=\{t: 0 \leqq t \leqq 1\}$, then for $p \neq 0, \sup _{t \in J} \rho\left(F_{p}(t), F_{0}(t)\right) \geqq 1 / 4$. That is, if $p \neq 0$, then $F_{p} \oplus \mathcal{O}\left(F_{0}, J, \epsilon\right)$ for any $\epsilon, 0<\epsilon<1 / 4$.

Actually the continuity of the mapping $H$ in the $\mathcal{O}$-topology on $W$ is closely related to the uniqueness of solutions of (1), as is shown in the following theorem.

ThEOREM 5. (A) If the solutions of (1) are unique, then the mapping $H: W \rightarrow W$ is continuous in the $\theta$-topology on $W$.

(B) $H$ is continuous in the $\Theta$-topology if and only if the O-topology and the Th-topology agree on $W$.

(C) If $H$ is continuous in the $\Theta$-topology, then the extension $G$ : $(K, \mathcal{F})$ $\rightarrow(K, \mathcal{O})$ is continuous in the given topologies.

It should be noted that Theorem 5 (A) is the classical statement of continuity with respect to initial conditions for solutions of (1).

An interesting question, for which the answer is apparently not known, is whether the following is true: " $H$ is continuous in the Q-topology on $W$ if and only if the solutions of (1) are unique."

7. The classification problem. Theorem 4 now gives a new context for phrasing the problem: "What kind of sets are sections of integral funnels?" We have shown that every differential equation (1) gives rise to a bicontinuous injection $G$ of $K$ into $H$. The following question now arises: "Is every bicontinuous injection $G$ of $K$ into $H$ generated by the integral funnels of some differential equation of the type (1) on $W$ ?" The following example gives a negative answer.

Let $W=R^{1}$ and

$$
F_{p}(t)= \begin{cases}p+(\operatorname{sgn} p) t^{2} / 4, & \text { if } p \neq 0 \\ \left\{t^{2} / 4,-t^{2} / 4\right\}, & \text { if } p=0\end{cases}
$$

The mapping $H: p \rightarrow F_{p}$ is a homeomorphism, but it is not generated by the integral funnels of a differential equation since $F_{0}(t)$ is not connected for $t \neq 0$, which is contrary to Kneser's theorem [7]. 


\section{BIBLIOGRAPHY}

1. E. A. Barbašin, On the theory of general dynamical systems, Uč. Zap. Moskov. Gos. Univ. 135 Matematika, Tom II, (1948), 110-133. (Russian)

2. M. Fukuhara, Sur les systèmes des equations différentielles ordinaires, Proc. Imp. Acad. Japan 4 (1928), 448-449.

3. - Sur les systèmes d'équations différentielles on dinaires. II, Japan J. Math. 6 (1930), 269-299.

4. F. Hausdorff, Set theory, Chelsea, New York, 1957.

5. E. Kamke, Zur Theorie der Systeme gewöhnlicher Differentialgleichungen. II, Acta Math. 58 (1932), 57-85.

6. J. L. Kelley, General topology, Van Nostrand, New York, 1955.

7. H. Kneser, Über die Lösungen eines Systemes gewöhnlicher Differentialgleichungen das der Lipschitzschen Bedingung nicht genügt, S.-B. Preuss. Akad. Wiss. Phys.-Math. K1. (1923), 121-174.

8. M. I. Minkevič, The theory of integral funnels in generalized dynamical systems without a hypothesis of uniqueness, Dokl. Akad. Nauk SSSR (N.S.) 59 (1948), 1049-1052. (Russian)

9. - Theory of integral funnels in dynamical systems without uniqueness, Uč. Zap. Moskov. Gos. Univ. 135, Matematika, Tom II, (1948), 134-151. (Russian)

10. E. O. Roxin, Axiomatic theory of control systems, RIAS Tech. Rep. No. 62-16, 1962.

11. R. L. Wilder, Topology of manifolds, Amer. Math. Soc. Colloq. Publ. Vol. 32, Amer. Math. Soc., Providence, R. I., 1949.

HARVARD UNIVERSITY 